\title{
A Linear-Time Algorithm for Bend-Optimal Orthogonal Drawings of Biconnected Cubic Plane Graphs
} (Extended Abstract)

\author{
Shin-ichi Nakano and Makiko Yoshikawa \\ Gunma University, Kiryu 376-8515, Japan \\ nakano@cs.gunma-u.ac.jp
}

\begin{abstract}
An orthogonal drawing of a plane graph $G$ is a drawing of $G$ with the given planar embedding in which each vertex is mapped to a point, each edge is drawn as a sequence of alternate horizontal and vertical line segments, and any two edges do not cross except at their common end. Observe that only a planar graph with the maximum degree four or less has an orthogonal drawing. The best known algorithm to find an orthogonal drawing runs in time $O\left(n^{7 / 4} \sqrt{\log n}\right)$ for any plane graph with $n$ vertices. In this paper we give a linear-time algorithm to find an orthogonal drawing of a given biconnected cubic plane graph with the minimum number of bends.
\end{abstract}

\section{Introduction}

An orthogonal drawing of a plane graph $G$ is a drawing of $G$ with the given planar embedding in which each vertex is mapped to a point, each edge is drawn as a sequence of alternate horizontal and vertical line segments, and any two edges do not cross except at their common end. Orthogonal drawings have attracted much attention due to its numerous practical applications in circuit schematics, etc. [BLV93 K96 T87]. In particular, we wish to find an orthogonal drawing with the minimum number of bends.

For a given planar graph $G$, if it is allowed to choose its planar embedding, then finding an orthogonal drawing of $G$ with the minimum number of bends is NP-complete[GT94]. However, Tamassia [T87] and Garg and Tamassia [GT96] presented algorithms which find an orthogonal drawing of a given plane graph $G$ with the minimum number of bends in $O\left(n^{2} \log n\right)$ and $O\left(n^{7 / 4} \sqrt{\log n}\right)$ time respectively unless it is allowed to choose its planar embedding, where $n$ is the number of vertices in $G$. They reduce the minimum-bend orthogonal drawing problem to a minimum cost flow problem. On the other hand, several lineartime algorithms are known for finding an orthogonal drawing of a plane graph with a presumably small number of bends[K96], and for 3-connected cubic plane graphs a linear-time algorithm is known for finding an orthogonal drawing with the minimum number of bends[RNN99]. Observe that only a planar graph with the maximum degree four or less has an orthogonal drawing.

J. Marks (Ed.): GD 2000, LNCS 1984, pp. 296-307 2001.

(C) Springer-Verlag Berlin Heidelberg 2001 


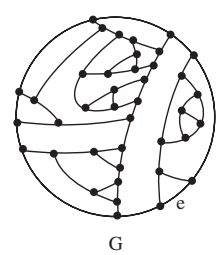

(a)

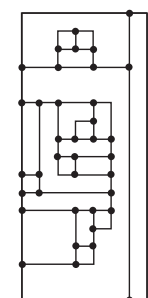

(b)

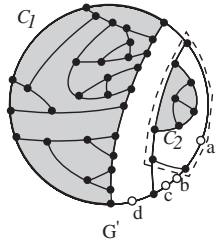

(c)

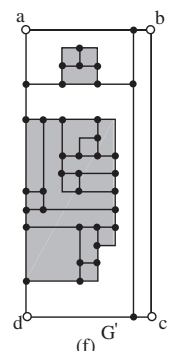

(d)

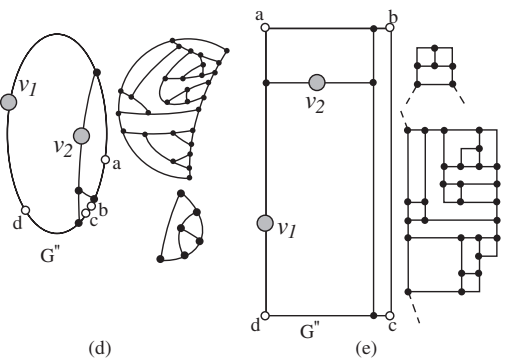

Fig. 1. A plane graph and its orthogonal drawing.

In this paper, generalizing the result in RNN99], we give a linear-time algorithm to find an orthogonal drawing of a biconnected cubic plane graph with the minimum number of bends.

An orthogonal drawing in which there is no bend and each face is drawn as a rectangle is called a rectangular drawing. Given a plane graph $G$ such that every vertex has degree either two or three, in linear-time we can find a rectangular drawing of $G$ whenever such a graph has a rectangular drawing KH94RNN96, RNN00. The key idea of our algorithm is to reduce the orthogonal drawing problem to the rectangular drawing problem.

An outline of our algorithm is illustrated in Fig. 1 Given a plane graph $G$ as shown in Fig. 1(a), we first find a tree structure among some cycles in $G$, then by analyzing the tree structure we put four dummy vertices $a, b, c$ and $d$ of degree two on the outer boundary of $G$, and let $G^{\prime}$ be the resulting graph. The four dummy vertices are drawn by white circles in Fig. 1(c). We then contract each of some cycles $C_{1}, C_{2}, \cdots$ and their insides (shaded in Fig. 1(c)) into a single vertex as shown in Fig. 1(d) so that the resulting graph $G^{\prime \prime}$ has a rectangular drawing as shown in Fig. 1(e). We also find orthogonal drawings of those cycles $C_{1}, C_{2}, \cdots$ and their insides recursively (See Figs. 1(d) and (e)). Patching the obtained drawings, we get an orthogonal drawing of $G^{\prime}$ as shown in Fig. 11(f). Replacing the dummy vertices $a, b, c$ and $d$ in the drawing of $G^{\prime}$ with bends, we finally obtain an orthogonal drawing of $G$ as shown in Fig. 1(b).

The rest of the paper is organized as follows. Section 2 gives some definitions and presents a known result. Section 3 shows a tree structure among some cycles in $G$. Section 4 presents an algorithm to find an orthogonal drawing with the minimum number of bends. 


\section{Preliminaries}

Let $G$ be a connected graph with $n$ vertices. An edge connecting vertices $x$ and $y$ is denoted by $(x, y)$. The degree of a vertex $v$ is the number of neighbors of $v$ in $G$. If every vertex of $G$ has degree three, then $G$ is called a cubic graph. The connectivity $\kappa(G)$ of a graph $G$ is the minimum number of vertices whose removal results in a disconnected graph or a single-vertex graph $K_{1}$. We say that $G$ is $k$-connected if $\kappa(G) \geq k$.

A graph is planar if it can be embedded in the plane so that no two edges intersect geometrically except at a vertex to which they are both incident. A plane graph is a planar graph with a fixed planar embedding. A plane graph divides the plane into connected regions called faces. We regard the contour of a face as a clockwise cycle formed by the edges on the boundary of the face. We denote the contour of the outer face of graph $G$ by $C_{o}(G)$.

For a simple cycle $C$ in a plane graph $G$, we denote by $G(C)$ the plane subgraph of $G$ inside $C$ (including $C$ ). We say that cycles $C_{1}$ and $C_{2}$ in a plane graph $G$ are independent if $G\left(C_{1}\right)$ and $G\left(C_{2}\right)$ have no common vertex. Cycles $C_{1}$ and $C_{2}$ are vertex-disjoint if $C_{1}$ and $C_{2}$ have no common vertex. An edge which is incident to exactly one vertex of a simple cycle $C$ and located outside of $C$ is called a leg of the cycle $C$, and the vertex on $C$ to which the leg is incident is called a leg-vertex of $C$. A simple cycle with exactly $k$ legs is called a $k$-legged cycle. For $k$-legged cycle $C$ the $k$ subpaths of $C$ dividing $C$ at the $k$ leg-vertices are called the contour paths of $C$.

An orthogonal drawing of a plane graph $G$ is a drawing of $G$ with the given planar embedding in which each vertex is mapped to a point, each edge is drawn as a sequence of alternate horizontal and vertical line segments, and any two edges do not cross except at their common end. A point where an edge changes its direction in a drawing is called a bend. We denote by $b(G)$ the minimum number of bends for orthogonal drawings of $G$. An orthogonal drawing of $G$ with exactly $b(G)$ bends is bend-optimal.

A rectangular drawing of a plane graph $G$ is a drawing of $G$ such that each edge is drawn as a horizontal or vertical line segment, and each face is drawn as a rectangle. Thus a rectangular drawing is an orthogonal drawing in which there is no bend and each face is drawn as a rectangle. The drawing of $G^{\prime \prime}$ in Fig. 1(e) is a rectangular drawing. The drawing of $G^{\prime}$ in Fig. प(f) is not a rectangular drawing, but is an orthogonal drawing. In any rectangular drawing $D$ of $G$, the four corners of the rectangle corresponding to $C_{o}(G)$ are vertices of degree two on $C_{o}(G)$. We call these four vertices the corner vertices of $D$. The following result on rectangular drawings is known.

Lemma 1. Let $G$ be a connected plane graph such that every vertex has degree either two or three, and let $a, b, c, d$ be four designated vertices of degree two on $C_{o}(G)$. Then $G$ has a rectangular drawing with the corner vertices $a, b, c, d$ if and only if $G$ has none of the following three types of simple cycles [T84]:

(r1) 1-legged cycles,

(r2) 2-legged cycles which contain at most one designated vertex of degree two, and 
(r3) 3-legged cycles which contain no designated vertex of degree two.

Furthermore one can check in linear time whether $G$ satisfies the condition above, and if $G$ does then one can find a rectangular drawing of $G$ in linear time [RNN96 RNN00].

\section{Genealogical Tree}

Let $G$ be a biconnected cubic plane graph. For a pair of distinct cycles $C_{a}$ and $C_{d}$ in $G, C_{d}$ is called a descendant-cycle of $C_{a}$ if (i) $C_{d}$ is either 2- or 3-legged cycle, and (ii) $G\left(C_{d}\right)$ is a proper subgraph of $G\left(C_{a}\right)$. Note that since $G$ is biconnected there is neither 0- nor 1-legged cycle except the only 0-legged cycle $C_{o}(G)$. Now we choose an edge $e=(x, y)$ on $C_{o}(G)$, and replace $e$ with two edges $(x, z)$ and $(z, y)$. Let $G^{\prime}$ be the resulting plane graph. (Note that, for $G-e$, that is a plane subgraph of $G$ obtained from $G$ by deleting $e, C_{o}(G-e)$ is a 2-legged cycle of $G^{\prime}$, however, $C_{o}(G-e)$ is not a 2-legged cycle of $G$.) Let $D_{e}\left(C_{o}\right)=\{C \mid C$ is a descendant cycle of $C_{o}\left(G^{\prime}\right)$ not containing $\left.z\right\}$. A cycle $C_{c}$ in $D_{e}\left(C_{o}\right)$ is called a child-cycle of $C_{o}\left(G^{\prime}\right)$ (with respect to edge $e$ ) if $C_{c}$ is not located inside of any other cycle in $D_{e}\left(C_{o}\right)$. Since $G$ is a biconnected cubic plane graph, $C_{o}\left(G^{\prime}\right)$ has exactly one child-cycle $C_{o}(G-e)$ (with respect to edge $e$ ). (See Fig 2 2 ) Then, recursively, for each child-cycle $C_{c}$ we define its child-cycle as follows. We have the following two cases.

Case 1: $C_{c}$ is a 2-legged cycle.

Choose a leg-vertex of $C_{c}$ as $z$. Let $D_{z}\left(C_{c}\right)=\{C \mid C$ is a descendant cycle of $C_{c}$ not containing $\left.z\right\}$. A cycle $C_{c c}$ in $D_{z}\left(C_{c}\right)$ is called a child-cycle of $C_{c}$ (with respect to $z$ ) if $C_{c c}$ is not located inside of any other cycle in $D_{z}\left(C_{c}\right)$. Since $G$ is a biconnected cubic plane graph, $C_{c}$ has at most one 3-legged child-cycle. $\left(C_{c}\right.$ has no 3-legged child-cycle if $G(C)$ has an inner face $F$ containing the two leg-vertices, and $C_{c}$ has exactly one 3-legged child-cycle otherwise.)

Case 2: Otherwise, $C_{c}$ is a 3-legged cycle.

Let $D\left(C_{c}\right)$ be the set of all descendant cycles of $C_{c}$. A cycle $C_{c c}$ in $D\left(C_{c}\right)$ is called a child-cycle of $C_{c}$ if $C_{c c}$ is not located inside of any other cycle in $D\left(C_{c}\right)$.

In both cases above all child-cycles of $C_{c}$ are independent each other.

By the definition above we can find child-cycles of each child-cycle recursively, and eventually we get a (hierarchical) tree structure of cycles in $G$ represented by a "genealogical tree" $T_{g}$, as shown in Fig 2. Because of the choices for $e$ and $z, T_{g}$ may have some variations. We choose an arbitrary (but fixed) one as $T_{g}$.

Using a method similar to one in RNN96 RNN99 RNN00], in linear time one can find such a tree structure $T_{g}$ among cycles by traversing the contour of each face a constant number of times.

Now we observe the following. In any orthogonal drawing of $G$, every cycle $C$ in $G$ has at least four convex corners, i.e., polygonal vertices of inner angle $90^{\circ}$. Since $G$ is cubic, such a corner must be a bend if it is not a leg-vertex of $C$. Thus we have the following facts for any orthogonal drawing of $G$. 


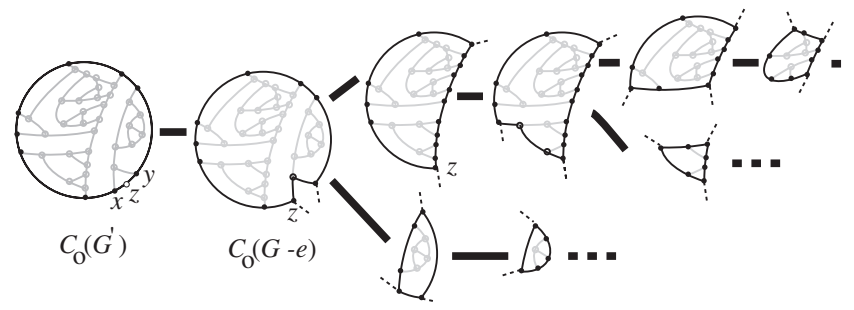

Fig. 2. cycles in $G^{\prime}$ and a genealogical tree $T_{g}$.

Fact 1 At least four bends must appear on $C_{o}(G)$.

Fact 2 At least two bend must appear on each 2-legged cycle in $G$.

Fact 3 At least one bend must appear on each 3-legged cycle in $G$.

\section{Orthogonal Drawing}

In this section we give a linear-time algorithm to find a bend-optimal orthogonal drawing of a biconnected cubic plane graph. Assume that we have a genealogical tree $T_{g}$ of a biconnected cubic plane graph $G$. We need some definitions.

Let $C$ be a 2-legged cycle with the two leg-vertices $x$ and $y$, and $P_{1}$ and $P_{2}$ be the clockwise contour paths from $x$ to $y$ and from $y$ to $x$, respectively. A bend-optimal orthogonal drawing $D$ of $G(C)$ is feasible for $\left(P_{1}, P_{1}\right)$ if none of the following four open halflines intersects $D$. (See Fig. 3)(a). Intuitively $D$ needs two convex bends on $P_{1}$.)

the vertical open halfline with the upper end at $x$.

the horizontal open halfline with the left end at $x$.

the vertical open halfline with the lower end at $y$.

the horizontal open halfline with the left end at $y$.
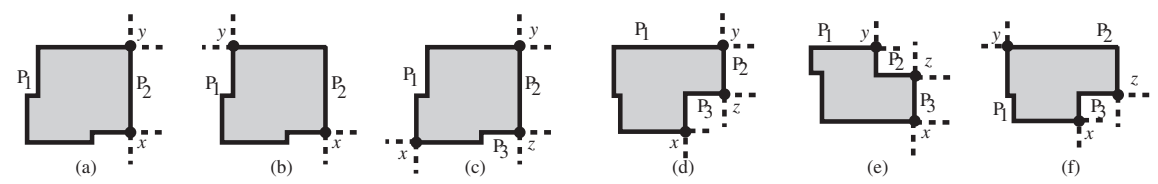

Fig. 3. Illustration for feasible drawings.

Also, a bend-optimal orthogonal drawing $D$ of $G(C)$ is feasible for $\left(P_{1}, P_{2}\right)$ if none of the four open halflines depicted in dashed lines in Fig. [3(b) intersects $D$.

Let $C$ be a 3-legged cycle with the three leg-vertices $x, y$ and $z$ appearing clockwise in this order, and $P_{1}, P_{2}$ and $P_{3}$ be the clockwise contour path from $x$ to $y$, from $y$ to $z$, and from $z$ to $x$, respectively. A bend-optimal orthogonal drawing $D$ of $G(C)$ is feasible for $\left(P_{1}\right)$ if none of the six open halflines depicted in dashed lines in Fig. B( $\mathrm{B}$ ) intersects $D$. Similarly, we define feasible orthogonal drawings for $\left(P_{1}, P_{1},-P_{3}\right),\left(P_{1}, P_{1},-P_{2}\right)$ and $\left(P_{1}, P_{2},-P_{3}\right)$.(See Fig. [3(d)-(f).) 
Now, for each cycle $C \neq C_{o}(G)$ corresponding to a vertex in $T_{g}$, we determine whether $G(C)$ has each type of feasible drawings by a bottom-up computation on $T_{g}$. For the bottom-up computation we also compute a set $S_{C}$ of vertexdisjoint cycles in $G(C)$ consisting of $\ell_{2}$ 2-legged cycles and $\ell_{3}$ 3-legged cycles for some $\ell_{2}$ and $\ell_{3}$. Thus $b(G(C)) \geq 2 \cdot \ell_{2}+\ell_{3}$ by Facts 3.2 and 3.3. We then show that $G(C)$ always has at least one feasible drawing using $2 \cdot \ell_{2}+\ell_{3}$ bends. Thus $b(G(C))=2 \cdot \ell_{2}+\ell_{3}$ holds.

In the bottom-up computation we classify each contour path of each cycle as either 0 -, 1-, or 2-corner path. Intuitively $k$-corner path has a chance to have $k$ convex bends. And we define $P_{1} P_{2}$-strain by those corner paths as follows. Let

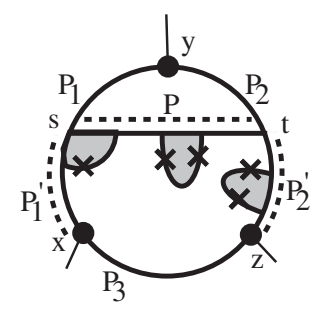

Fig. 4. Illustration for $P_{1} P_{2}$-strain.

$x, y, z$ be the three leg-vertices of a 3-legged cycle $C, P_{1}$ and $P_{2}$ be the clockwise contour paths from $x$ to $y$ and $y$ to $z$, respectively. Assume that $s$ and $t$ are vertices on $P_{1}$ and $P_{2}$, respectively, and let $P_{1}^{\prime}$ be the subpath of $P_{1}$ from $x$ to $s$, and $P_{2}^{\prime}$ be the subpath of $P_{2}$ from $t$ to $z$. If (i) there is a path $P$ from $s$ to $t$ such that the left side of $P$ is an inner face of $G(C)$, and (ii) $G(C)$ has no child cycle having 1- or 2-corner path on $P, P_{1}^{\prime}$ or $P_{2}^{\prime}$, then the path consisting of $P_{1}^{\prime}, P, P_{2}^{\prime}$ are called $P_{1} P_{2}$-strain. An example is illustrated in Fig. 4. Intutively, we have only two chance to turn right at $s$ and $t$ on $P_{1} P_{2}$-strain from $x$ to $z$.

In the bottom-up computation we show that the following conditions $(\mathrm{c} 1)-$ (c9) hold.

(c1) Any cycle $C$ has at least one 1- or 2-corner path.

(c2) No cycle in $S_{C}$ contains any edge on any 0-corner path of $C$.

(c3) For any 2-legged cycle $C$ if $C$ has a 1-corner path $P_{1}$, then $G(C)$ has a set $S_{C}^{\prime}$ of vertex-disjoint cycles containing no edge on $P_{1}$ and consisting of $\ell_{2}^{\prime}$ 2-legged cycles and $\ell_{3}^{\prime}$ 3-legged cycles such that $2 \cdot \ell_{2}^{\prime}+\ell_{3}^{\prime}=b(G(C))-1$.

(c4) For any 2-legged cycle $C$ if $C$ has a 0 -corner path $P_{1}$, then the other contour path $P_{2}$ is a 2-corner path, and $G(C)$ has an orthogonal drawing feasible for $\left(P_{2}, P_{2}\right)$.

(c5) For any 3-legged cycle $C$ if $C$ has a 1-corner path $P_{1}$, then $G(C)$ has a set $S_{C}^{\prime}$ of vertex-disjoint cycles containing no edge on $P_{1}$, and consisting of $\ell_{2}^{\prime}$ 2-legged cycles and $\ell_{3}^{\prime}$ 3-legged cycles such that $2 \cdot \ell_{2}^{\prime}+\ell_{3}^{\prime}=b(G(C))-1$.

(c6) For any 3-legged cycle $C$ if $C$ has a 1- or 2-corner path $P_{1}$, then $G(C)$ has an orthogonal drawing feasible for $\left(P_{1}\right)$.

(c7) For any 3-legged cycle $C$ if $C$ has a 2-corner path $P_{1}$ and no $P_{1} P_{2}$-strain , then $G(C)$ has an orthogonal drawing feasible for $\left(P_{1}, P_{1},-P_{3}\right)$, 
(c8) For any 3-legged cycle $C$ if $C$ has a 2-corner path $P_{1}$ and no $P_{3} P_{1}$-strain, then $G(C)$ has an orthogonal drawing feasible for $\left(P_{1}, P_{1},-P_{2}\right)$,

(c9) For any 3-legged cycle $C$ if $C$ has 1-corner paths $P_{1}$ and $P_{2}$, and no $P_{1} P_{2^{-}}$ strain, then $G(C)$ has an orthogonal drawing feasible for $\left(P_{1}, P_{2},-P_{3}\right)$.

Now we explain the bottom-up computation in the following four cases.

Case 1: $C$ is a 2-legged cycle having no child-cycle.

Let $x, y$ be the two leg-vertices of $C$, let $P_{1}$ and $P_{2}$ be the clockwise contour paths from $x$ to $y$ and from $y$ to $x$, respectively. Now $G(C)=C$, since for any 2-legged cycle $C$ if $G(C)$ has an edge in proper inside of $C$ then $C$ always has a child-cycle.

Computation for $\boldsymbol{S}_{\boldsymbol{C}}$ : Set $S_{C}=\{C\}$. By Fact 3.2 any orthogonal drawing of $G(C)$ has at least two bends.

Feasible drawings: By introducing two bends on $P_{1}$, we can easily construct an orthogonal drawing of $G(C)$ feasible for $\left(P_{1}, P_{1}\right)$. Similarly we can construct orthogonal drawings of $G(C)$ feasible for $\left(P_{2}, P_{2}\right)$ and $\left(P_{1}, P_{2}\right)$, respectively. Thus $G(C)$ has each type of feasible orthogonal drawings.

Classification and proof for (c1)-(c9): In this case every contour path of $C$ is classified as a 2-corner paths. Conditions (c1)-(c4) hold since every contour path of $C$ is 2-corner, and (c5)-(c9) hold since $C$ is not a 3-legged cycle.

Case 2: $C$ is a 3-legged cycle having no child-cycle.

Let $x, y, z$ be the three leg-vertices of $C$, let $P_{1}, P_{2}, P_{3}$ be the clockwise contour path from $x$ to $y$, from $y$ to $z$, and from $z$ to $x$, respectively. Now if we remove all edges on $C$ from $G(C)$, then either $G(C)=C$ or the remaining edges induce a connected graph containing at least one vertex on each $P_{1}, P_{2}, P_{3}$, since otherwise $C$ has a child-cycle, a contradiction.

Computation for $\boldsymbol{S}_{C}$ : Set $S_{C}=\{C\}$. By Fact 3.3 any orthogonal drawing of $G(C)$ has at least one bend.

Feasible drawings: Construct a new graph $G^{\prime}$ from $G(C)$ by adding one dummy vertices $v$ on $P_{1}$. Now the resulting graph $G^{\prime}$ has no bad cycle (since $G$ has no child-cycle) with respect to corner vertices $x, v, y, z$, and then $G^{\prime}$ has a rectangular drawing with the corner vertices $x, v, y, z$. The rectangular drawing is also an orthogonal drawing of $G(C)$ feasible for $\left(P_{1}\right)$ using exactly one bend (corresponding to $v$ ). Similarly we can easily construct orthogonal drawings of $G(C)$ feasible for $\left(P_{2}\right)$ and $\left(P_{3}\right)$.

Now $G(C)$ has no orthogonal drawing feasible for $\left(P_{1}, P_{1},-P_{2}\right)$, since it needs at least two bends only on $P_{1}$. Similarly $G(C)$ has no orthogonal drawing feasible for $\left(P_{i}, P_{j},-P_{k}\right)$ for any $i, j, k \in\{1,2,3\}$.

Classification and proof for (c1)-(c9): In this case every contour path of $C$ is classified as a 1-corner path. Conditions (c1),(c2) hold since every contour path of $C$ is 1-corner, (c3),(c4) hold since $C$ is not a 2-legged cycle, (c5) holds by choosing $S_{C}^{\prime}=\phi$, (c6) holds since $G(C)$ has orthogonal drawings feasible for $\left(P_{1}\right),\left(P_{2}\right),\left(P_{3}\right)$, respectively, as mentioned above, and $(\mathrm{c} 7)-(\mathrm{c} 9)$ hold since $G(C)$ has no 2-corner path.

Case 3: $C$ is a 2-legged cycle having one or more child-cycles.

Let $x, y$ be the two leg-vertices of $C$, and let $P_{1}$ and $P_{2}$ be the clockwise contour paths from $x$ to $y$ and from $y$ to $x$, respectively. If $G(C)$ has an inner 
face containing $x$ and $y$, then $C$ has no 3-legged child-cycle, otherwise, $C$ has exactly one 3-legged child-cycle, which contains exactly one leg-vertices of $C$. Thus $C$ has at most one 3-legged child-cycle.

Let $C_{1}, C_{2}, \cdots, C_{\ell}$ be the child-cycle of $C$. Assume that for $C_{i}, 1 \leq i \leq l$, we already have $S_{C_{i}}$, we know whether $G\left(C_{i}\right)$ has each type of feasible drawings, and conditions (c1)-(c9) holds. We have the following four subcases. Proofs for (c1)-(c9) are omitted.

Case 3(a): $C$ has no child-cycle having a 1- or 2- corner path on $C$.

Computation for $\boldsymbol{S}_{C}$ : Condition (c2) means that no cycle in $S_{C_{1}}, S_{C_{2}}, \cdots, S_{C_{\ell}}$ contains any edge on $C$. Also since $G$ is cubic, $C$ is vertex-disjoint to any cycle in $S_{C_{1}}, S_{C_{2}}, \cdots, S_{C_{\ell}}$. Set $S_{C}=\{C\} \cup S_{C_{1}} \cup S_{C_{2}} \cup \cdots \cup S_{C_{\ell}}$. Thus we need to introduce two new bends.

Feasible drawings: We first consider whether $G(C)$ has an orthogonal drawing feasible for $\left(P_{1}, P_{1}\right)$. Construct a new graph from $G(C)$ by adding two dummy vertices $v, w$ on $P_{1}$ but not on any child cycle of $C$. Then contract each $G\left(C_{1}\right), G\left(C_{2}\right), \cdots, G\left(C_{\ell}\right)$ to vertices $v_{1}, v_{2}, \cdots, v_{\ell}$, respectively. See Figs. 5 (a) and (b). Now the resulting graph is a cycle and has a rectangular drawing $D$ with the corner vertices $x, v, w, y$. See Fig. 5)(c). Next, if $C$ has a 3-legged child-cycle, say $C^{\prime}$, then find an orthogonal drawing of $G\left(C^{\prime}\right)$ feasible for $\left(P^{\prime}\right)$ where $P^{\prime}$ is the contour path of $C^{\prime}$ not on $C$, in a recursive manner. By conditions (c1) and (c6) $G\left(C^{\prime}\right)$ always has such a drawing. Next, find an orthogonal drawing of each 2-legged child-cycle $G\left(C_{i}\right)$ feasible for $\left(P_{i}^{\prime \prime}, P_{i}^{\prime \prime}\right)$ where $P_{i}^{\prime \prime}$ is the contour path of $C_{i}$ not on $C$, in a recursive manner. By condition (c4) $G(C)$ always has such a drawing. Finally patch the drawings of $G\left(C_{1}\right), G\left(C_{2}\right), \cdots, G\left(C_{\ell}\right)$ into $D$. See Fig. 5(d). The patching for 2- and 3-legged child-cycles always works correctly as shown in Fig. 6] and Fig. 7. Thus we can construct an orthogonal drawing of $G(C)$ feasible for $\left(P_{1}, P_{1}\right)$. Similarly we can construct orthogonal drawings feasible for $\left(P_{2}, P_{2}\right)$ and $\left(P_{1}, P_{2}\right)$, respectively.

Classification: In this case every contour path of $C$ is classified as a 2-corner path.

Case 3(b): $C$ has exactly one child-cycles having a 1- or 2- corner path on $C$, and the child-cycle is a 2-legged cycle.

Computation for $S_{C}$ : Let $C_{1}$ be the 2-legged child-cycle having a corner path on $C$. We consider two cases. If $C_{1}$ has a 2-corner path on $C$, then set $S_{C}=S_{C_{1}} \cup$ $S_{C_{2}} \cup \cdots \cup S_{C_{\ell}}$. In this case we do not need to introduce any new bends. If $C_{1}$ has a 1-corner path on $C$, then, by (c3), $G\left(C_{1}\right)$ has a set $S_{C_{1}}^{\prime}$ of vertex-disjoint cycles containing no edge on $C$, and consisting of $\ell_{2}^{\prime}$ 2-legged cycles and $\ell_{3}^{\prime}$ 3-legged cycles such that $2 \cdot \ell_{2}^{\prime}+\ell_{3}^{\prime}=b\left(G\left(C_{1}\right)\right)-1$. Condition (c2) means that no cycle in $S_{C_{2}}, S_{C_{3}}, \cdots, S_{C_{\ell}}$ contains any edge on $C$. Set $S_{C}=\{C\} \cup S_{C_{1}}^{\prime} \cup S_{C_{2}} \cup \cdots \cup S_{C_{\ell}}$. In this case we need to introduce one new bend.

Feasible drawings: Omitted. Similar to the previous case.

Classification: If $C_{1}$ has a 2-corner path on $P_{1}$, then $P_{1}$ is a 2-corner path and $P_{2}$ is a 0 -corner path. If $C_{1}$ has a 2 -corner path on $P_{2}$, then $P_{1}$ is a 0 -corner path and $P_{2}$ is a 2-corner path. If $C_{1}$ has a 1 -corner path on $P_{1}$, then $P_{1}$ is a 2-corner path and $P_{2}$ is a 1-corner path. (In this case we can add one new bend either on 


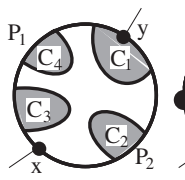

(a)

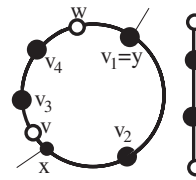

(b)

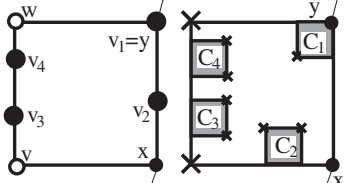

(c) (d)

Fig. 5. Illustration for Case 3(a).

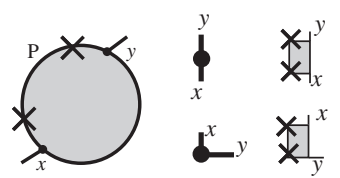

(a)

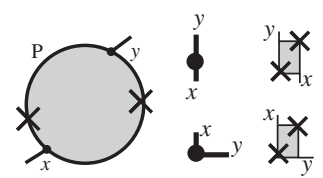

(b)

Fig. 6. Illustration for patchings.

$P_{1}$ or $P_{2}$.) If $C_{2}$ has a 1 -corner path on $P_{2}$, then $P_{1}$ is a 1 -corner path and $P_{2}$ is a 2-corner path.

Case 3(c): $C$ has exactly one child-cycles having a 1- or 2-corner path on $C$, and the child-cycle is a 3-legged cycle.

Let $C_{1}$ be the 3-legged child-cycle having a 1- or 2-corner path on $C$. Assume that $C_{1}$ shares $y$ with $C$ as a leg-vertex. Let $P_{11}$ be the contour path of $C_{1}$ on $P_{1}$ and $P_{12}$ be the contour path of $C_{1}$ on $P_{2}$.

Computation for $\boldsymbol{S}_{C}$ : We consider three cases.

If $C_{1}$ has a $P_{11} P_{12}$-strain, then set $S_{C}=\left\{C_{S}\right\} \cup S_{C_{1}} \cup S_{C_{2}} \cup \cdots \cup S_{C_{\ell}}$, where $C_{S}$ is the 3-legged cycle consisting of the $P_{11} P_{12}$-strain and the edges on $P_{1}$ and $P_{2}$ not contained in $C_{1}$. By the definition of strain and (c2), $C_{S}$ is vertex-disjoint to any cycle in $S_{C_{1}}$. In this casewe need to introduce one new bend for $C_{S}$. (See Figs. 8(a)-(d).)

Otherwise, if $C_{1}$ has no $P_{11} P_{12}$-strain and either (i) $P_{11}$ is a 2 -corner path, (ii) $P_{12}$ is a 2 -corner path or (iii) $P_{11}$ is a 1-corner path and $P_{12}$ is a 1-corner path, then set $S_{C}=S_{C_{1}} \cup S_{C_{2}} \cup \cdots \cup S_{C_{\ell}}$. In this case we do not need to introduce any new bends. (See Figs. $8(\mathrm{e})-(\mathrm{g})$.)

Otherwise, $C_{1}$ has no $P_{11} P_{12}$-strain, and either (i) $P_{11}$ is a 1-corner path and $P_{12}$ is a 0 -corner path, or (ii) $P_{11}$ is a 0-corner path and $P_{12}$ is a 1-corner path. By (c5) $G\left(C_{1}\right)$ has a set $S_{C_{1}}^{\prime}$ of vertex-disjoint cycles containing no edge on $C$,

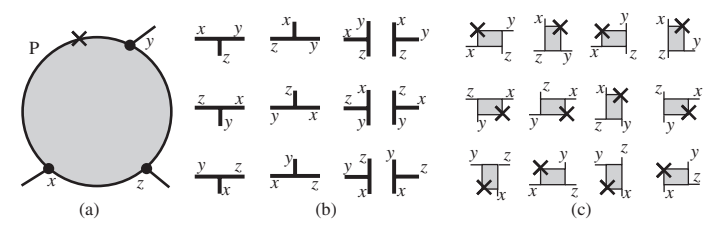

Fig. 7. Illustration for patchings. (Rotated cases are omitted.) 
and consisting of $\ell_{2}^{\prime}$ 2-legged cycles and $\ell_{3}^{\prime}$ 3-legged cycles such that $2 \cdot \ell_{2}^{\prime}+\ell_{3}^{\prime}=$ $b\left(G\left(C_{1}\right)\right)-1$. Set $S_{C}=\{C\} \cup S_{C_{1}}^{\prime} \cup S_{C_{2}} \cup \cdots \cup S_{C_{\ell}}$. Thus in this case we need to introduce one new bend. (See Figs. 8 (a) $-(d)$.)

Feasible drawings: Omitted. Similar to the previous case.

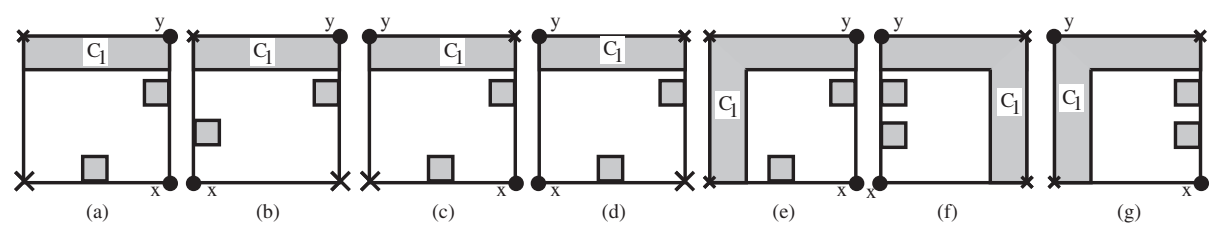

Fig. 8. Illustration for Case 3(c).

Classification: If either (i) $P_{11}$ is a 2-corner path and $C_{1}$ has no $P_{11} P_{12}$-strain, (ii) $P_{11}$ is a 1 - or 2-corner path and $C_{1}$ has a $P_{11} P_{12}$-strain, or (iii) $P_{11}$ is a 1-corner path, $P_{12}$ is a 0 -corner path and $C_{1}$ has no $P_{11} P_{12}$-strain, then $P_{1}$ is a 2-corner path. (See Figs. 8(e),(a),(a), respectively.) Otherwise if (i) $P_{11}$ is a 1-corner path, $P_{12}$ is a 1 -corner path and $C_{1}$ has no $P_{11} P_{12}$-strain, (ii) $P_{11}$ is a 0-corner path, $P_{12}$ is a 1 - or 2 -corner path and $C_{1}$ has a $P_{11} P_{12}$-strain, or (iii) $P_{11}$ is a 0 -corner path, $P_{12}$ is a 1 -corner path and $C_{1}$ has no $P_{11} P_{12}$-strain, then $P_{1}$ is a 1-corner path. (See Figs. $8(\mathrm{~g}),(\mathrm{c}),(\mathrm{c})$, respectively.) Otherwise, $P_{11}$ is a 0 -corner path, $P_{12}$ is a 2-corner path and $C_{1}$ has no $P_{11} P_{12}$-strain, then $P_{1}$ is a 0-corner path. (See Fig. $8(f)$.) Classify $P_{2}$ similarly.

Case 3(d): $C$ has two or more child-cycles having a 1- or 2- corner path on $C$. Omitted

Case 4: $C$ is a 3-legged cycle having one or more child-cycles.

Let $x, y, z$ be the three leg-vertices of $C$, and let $P_{1}, P_{2}, P_{3}$ be the clockwise contour path from $x$ to $y$, from $y$ to $z$, and from $z$ to $x$, respectively.

Computation for $\boldsymbol{S}_{C}$ : If $C$ has no child-cycle having a 1- or 2-corner path on $C$ then set $S_{C}=\{C\} \cup S_{C_{1}} \cup S_{C_{2}} \cup \cdots \cup S_{C_{\ell}}$. In this case we need to introduce one new bend. Otherwise set $S_{C}=S_{C_{1}} \cup S_{C_{2}} \cup \cdots \cup S_{C_{\ell}}$. In this case we do not need to introduce any new bend.

Feasible drawings: If $G(C)$ has no child-cycle having a 1- or 2-corner path on $C$ then $G(C)$ has orthogonal drawings feasible for $\left(P_{1}\right),\left(P_{2}\right),\left(P_{3}\right)$, respectively. (In this case we need to introduce one new bend.)

Otherwise, $G(C)$ has an orthogonal drawing feasible for $\left(P_{1}\right)$ if and only if $G(C)$ has a child-cycle having a 1- or 2-corner path on $P_{1}$. Similarly we can determine whether $G(C)$ has orthogonal drawings feasible for $\left(P_{2}\right)$ and $\left(P_{3}\right)$.

If $C$ has no child-cycle having a 1- or 2-corner path on $C$ then $G(C)$ has no orthogonal drawing feasible for $\left(P_{1}, P_{1},-P_{3}\right)$, since we have no chance to have two bend on $P_{1}$ even if we introduce one new bend on $P_{1}$.

$G(C)$ has an orthogonal drawing feasible for $\left(P_{1}, P_{1},-P_{3}\right)$ if and only if (i) $C$ has two child-cycle having a 1 - or 2-corner path on $P_{1}$, or $C$ has a child-cycle having a 2-corner path on $P_{1}$, and (ii) $C$ has no $P_{1} P_{2}$-strain. (Construction is omitted. See Figs. 9 and 10, ) 


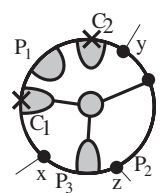

(a)

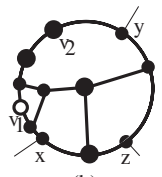

(b)

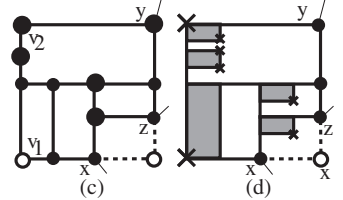

(d)

Fig. 9. Illustration for Case 4.

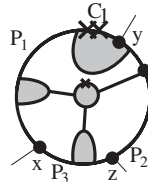

(a)

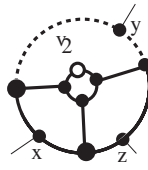

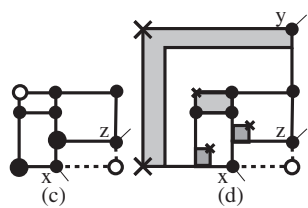

Fig. 10. Illustration for Case 4.

Classification: If $C$ has no child-cycle having a 1- or 2-corner path on $C$, then $P_{1}, P_{2}$ and $P_{3}$ are 1-corner paths. Otherwise, if either (i) $C$ has two or more child-cycles having a 1 - or 2 -corner path on $P_{1}$, or $C$ has a child-cycle having a 2-corner path on $P_{1}$, then $P_{1}$ is classified as a 2-corner path. Otherwise if $C$ has exactly one child-cycle having 1-corner path on $P_{1}$, then $P_{1}$ is classified as a 1-corner path. Otherwise $P_{1}$ is classified as a 0 -corner path. We classify $P_{2}$ similarly.

Now we give our algorithm to find a bend-optimal orthogonal drawing. Using a method similar to one in RNN96|RNN99|RNN00 the algorithm above runs in linear time.

\section{Algorithm Orthogonal-Draw $(G)$}

\section{begin}

1 Choose an edge $e$ on $C_{o}(G)$; Find a genealogical tree $T_{g}$;

2 Do the bottom-up computation;

3 Find minimal cycles having 1- or 2-corner path on $C_{o}\left(G^{\prime}\right)$ as many as possible;

4 Do the following until $G_{0}$ has exactly four vertices of degree two.

For each minimal 2-legged cycle $C$ having 2-corner path on $G_{0}$ replace $G(C)$ with a quadrangle containing two vertices of degree two on $G_{0}$.

For each minimal 2-legged cycle $C$ having 1-corner path on $G_{0}$ replace $G(C)$ with a vertex of degree two.

For each minimal 3-legged cycle $C$ having 1-corner path on $G_{0}$ replace $G(C)$ with a quadrangle containing one vertex of degree two on $G_{0}$.

Put vertices of degree two on the edge $e$.

5 Find maximal bad cycles $C_{1}, C_{2}, \cdots, C_{\ell}$;

6 Let $G^{\prime \prime}$ be the graph derived from $G^{\prime}$ by contracting each $G\left(C_{i}\right), i=$ $1,2, \cdots, \ell$ into a vertex $v_{i}$;

7 Find a rectangular drawing $D\left(G^{\prime \prime}\right)$ of $G^{\prime \prime}$;

8 For each $i=1,2, \cdots, \ell$, find a feasible orthogonal drawing $D\left(G\left(C_{i}\right)\right)$ of $G\left(C_{i}\right)$;

9 Patch the drawings $D\left(G\left(C_{i}\right)\right), i=1,2, \cdots, \ell$, into $D\left(G^{\prime \prime}\right)$ to get an orthogonal drawing of $G$; (See Figs. 1 (e) and (f).)

end. 
Theorem 1. The algorithm above find a bend-optimal orthogonal drawing of a biconnected cubic plane graph in linear time.

\section{References}

[BLV93] G. Di Battista, G. Liotta and F. Vargiu, Spirality of orthogonal representations and optimal drawings of series-parallel graphs and 3-planar graphs, Proc. of Workshop on Algorithms and Data structures, LNCS 709, Springer (1993) 151-162.

[GT94] A. Garg and R. Tamassia, On the computational complexity of upward and rectilinear planarity testing, Proc. of Graph Drawing'94, LNCS 894, Springer (1995) 286-297.

[GT96] A. Garg and R. Tamassia, A new minimum cost flow algorithm with applications to graph drawing, Proc. of Graph Drawing'96, LNCS 1190, Springer (1997) 201-226.

[K96] G. Kant, Drawing planar graphs using the canonical ordering, Algorithmica, 16 (1996) 4-32.

[KH94] G. Kant and X. He, Two algorithms for finding rectangular duals of planar graphs, Proc. of WG'93, LNCS 790, Springer (1994) 396-410.

[RNN96] M. S. Rahman, S. Nakano and T. Nishizeki, Rectangular grid drawings of plane graphs, Proc. of COCOON'96, LNCS 1090, Springer (1996) 92-105. Also, Computational Geometry: Theory and Applications, 10 (1998) 203220 .

[RNN99] M. S. Rahman, S. Nakano and T. Nishizeki, A linear algorithm for bendoptimal orthogonal drawings of triconnected cubic plane graphs, Journal of Graph Algorithms and Applications, 3 (1999) 31-62.

[RNN00] M. S. Rahman, S. Nakano and T. Nishizeki, Rectangular Drawings of Plane Graphs without Designated Corners, Proc. of COCOON'00, LNCS 1858, Springer (2000) 85-94.

[T87] R. Tamassia, On embedding a graph in the grid with the minimum number of bends, SIAM J. Comput., 16 (1987) 421-444.

[T84] C. Thomassen, Plane representations of graphs, (Eds.) J.A. Bondy and U.S.R. Murty, Progress in Graph Theory, Academic Press Canada (1984) 43-69. 